\title{
Innovative Skills for Better Critical Care Nursing in Egypt
}

\author{
Dr. Hanan Mohammed Mohammed \\ Assistant Professor of Medical-Surgical Nursing Department, Faculty of Nursing, Ain Shams University, Egypt
}

\begin{abstract}
Intensive care today is the composite of a cohort of critically ill patients nursed in one environment that facilitates the support of organs to maintain physiological normality. Egyptians had documented procedures resembling tracheostomies to treat airway obstruction from as early as 1500 BC. Critical care or ICU nursing is a specialization of nursing which deals specifically with patients experiencing high-dependency, life -threatening conditions. In Egypt, Since the 1950s, intensive care has grown into a specialty in its own right. Significant technological advances have allowed us to develop sophisticated ventilators, renal replacement therapy and cardiovascular monitoring. Intensive care units can now even be supervised via tele or remote ICU systems, providing surveillance and support to a large number of ICUs in distant or remote sites by a centralized multidisciplinary critical care team. In Egypt, modern technologies are in progress and development in the field of critical care management, especially in keeping records and databases for the patients. Egypt develop a technology solution and enterprise model that organizes the time slots for baby incubators and/or ICUs and keeps track of the vacant time slots.
\end{abstract}

Keywords: Critical Care, Egypt, Innovative, Project.

\section{Introduction}

Intensive care and its development is part of an evolutionary process in the general organization of hospital medical practice ${ }^{(1)}$. Intensive care today is the composite of a cohort of critically ill patients nursed in one environment that facilitates the support of organs to maintain physiological normality ${ }^{(2)}$. Although we think of intensive care as a modern concept, organ support dates back thousands of years. Egyptians had documented procedures resembling tracheostomies to treat airway obstruction from as early as $1500 \mathrm{BC}$, and Adhikari et al., ${ }^{(3)}$ had commenced a form of organ support by cannulating the airway to allow "air to be drawn into the lungs" one thousand years later.

Critical care or ICU nursing is a specialization of nursing which deals specifically with patients experiencing high-dependency, life -threatening conditions ${ }^{(4)}$. There are a range of ICU specialties where critical care nurses may work including surgical, trauma, coronary, medical, pediatric, burns, cardiothoracic and high risk nurseries ${ }^{(5)}$. Nurses in the ICU often work in conjunction with a multidisciplinary team of doctors, consultants, physiotherapists and various other specialists to ensure optimal patient care. Critical care nursing can be mentally and physically demanding with long hours (including night shifts), extensive patient handling and high-pressure conditions. Critical care nurses must be able to make sophisticated judgements quickly as patients in ICU are often unstable and can experience rapid physiological decline ${ }^{(6)}$.

The duties of a critical care nurse may include assisting physicians during procedures, checking patients' vital signs, taking blood samples, managing ventilation and life support equipment and ordering diagnostic tests ${ }^{(7)}$. A critical care nurse can also administer medication and provide patients with personal care, such as bathing and dressing. Critical care nurse may need to undertake family liaison duties, such as providing support and education to both the patient and the patient's family. When a patient is discharged from the ICU and transferred to another unit in the hospital, the critical nurse will liaise with the new team to ensure a safe transition ${ }^{(8)}$.

Florence Nightingale made a revolutionary step towards modern critical care during the Crimean War in the 1850 s by separating wounded soldiers depending on the severity of their injuries ${ }^{(4,9)}$. A key component to intensive care of a patient is the frequency and intensity of monitoring by a designated nurse, a system that Florence recognized by monitoring the sickest soldiers more regularly by more nurses. Although she remained unconvinced about germ theory, her emphasis on cleanliness had a significant impact on reducing the mortality of the soldiers ${ }^{(10)}$. Additionally, her innovative data collection relating to hospital acquired infections allowed comparisons between hospitals and instigated the evidence based practice that we continue today ${ }^{(11)}$.

In Egypt, Since the 1950s, intensive care has grown into a specialty in its own right. Significant technological advances have allowed us to develop sophisticated ventilators, renal replacement therapy and cardiovascular monitoring. Intensive care units can now even be supervised via tele or remote ICU systems, providing surveillance and support to a large number of ICUs in distant or remote sites by a centralized multidisciplinary critical care team ${ }^{(6)}$. 


\section{Critical Care Teams}

In this way, the role of intensive care has rapidly expanded over the last 20 years with critical care staff being involved not only in the care of critically ill patients within the ICU, but also of those on general wards before and after their critical illness and even following discharge ${ }^{(12,13)}$. Of particular interest, and the beneficiary of significant financial investment over the last decade, has been the intuitively beneficial process of reviewing and treating patients early on in their acute illness, in order to prevent further deterioration and death. Multidisciplinary teams, consisting of experienced staff trained in intensive care, have been developed internationally over the last 15 years to review such patients ${ }^{(14,15)}$.

The teams were assembled in response to increasing evidence suggesting that unexpected mortality and morbidity may be prevented by early recognition of deterioration and prompt resuscitation of sick patients ${ }^{(3,16)}$. The team, comprising of medical and nursing staff trained in critical care, replaced the traditional cardiac arrest team. The rapid response team generally consists of a physician, a nurse and a respiratory specialist and is summoned prior to the "code" or cardiac arrest team ${ }^{(6,17)}$.

The history of critical care teams in Egypt was also born from the need to provide a national approach for the management of at risk patients and the creation of "Outreach teams" to provide and support the care of sick patients on the ward. Three essential objectives of an Outreach team were ${ }^{(6)}$ :

- To avert admission by identifying patients who are deteriorating and instituting treatment early, or by ensuring timely admission to an area where they can be treated to ensure the best outcome.

- To support the continued recovery of previously critically ill patients discharged to the ward and after discharge from hospital.

- To share critical care expertise and experience.

\section{Critical Care Management}

The main obvious problem at state hospitals is the over-crowdedness. According to the Open Data for Egypt website, total number of public and central hospitals in Egypt is 263 hospitals serving a total population of around 91 million ${ }^{(17)}$. Meanwhile, modern technologies are in progress and development in the field of critical care management, especially in keeping records and databases for the patients. Egypt develop a technology solution and enterprise model that organizes the time slots for baby incubators and/or ICUs and keeps track of the vacant time slots ${ }^{(18)}$.

\section{1. Management Sciences for Health Project:}

It is focused on improving the Performance of Nurses in Upper Egypt. While the Egyptian health system is characterized by an extensive infrastructure, an adequate number of physicians and nurses, medical technology and medicines, and good access to care, there still in need to improve quality of care. The Improving the Performance of Hospital Nurses (IPHN) project works with the Ministry of Health (MOH) to address this challenge. The IPHN program is building the leadership and management capacity of government hospital nurses to enable the staff to improve infection control, basic nursing care, and communication ${ }^{(17)}$. The program is enabling nurses to adhere to national indicators for infection control included critical care nursing. The objective is to improve the services and health outcomes of hospitals and primary health care units in five Upper Egypt Governorates (Aswan, Luxor, Qena, Assiut, and Sohag) by improving the performance of hospital and primary health care nurses to lead and manage their teams to address their challenges and achieve measurable results in four focus areas: infection control, basic nursing care, communication between health care providers and patients, and a new emphasis on other primary health care service delivery challenges related to the primary health care package of services, particularly integrated family planning, reproductive health and maternal and child health services ${ }^{(19)}$.

\subsection{Egypt's Improving Performance of Nurses Project Helps Cardiac Patients in Qena General Hospital (Creditable Story):}

It is 2:00 a.m., and Arabey El Sayed has been transported from a village 5km away to the emergency department of Qena General Hospital with severe chest pain. He is a 75-year-old farmer, and is married with five grown children. Dr. Ahmad, the physician on duty, is assisted by Abeer and Shimaa, two nurses trained through the Improving Performance of Nurses (IPN) Project. They work together quickly to provide the patient with the necessary emergency care. A nurse administers the ECG, and the patient is diagnosed with myocardial infarction and moved to the Cardiac Care Unit. Fifteen days later, he is comfortable, stable and able to return home. The IPN project is funded by USAID and implemented by Management Sciences for Health in Aswan, Luxor and Qena Governorates in Upper Egypt. The project combines leadership development with quality improvement and collaborative learning approaches. Nurses learned how to share a common vision, identify a priority health challenge, analyze its root causes, and design and implement an action plan to address the 
challenge. They also applied the Work Climate Assessment (WCA) tool, and worked as a team to improve the work climate for better work relations and service quality ${ }^{(17,18)}$.

In an improved work climate, the Qena General Hospital team of nurses defined their cardiac care unit goal, presented their case to their managers (including the Hospital Manager), and implemented their action plan to reach their goals. As an indicator of their improved work climate, their WCA score increased from 2.43 to 4.8 out of 5.0, an achievement of $97.5 \%$ of their target. The nurse team improved cardiac care by increasing the number of nurses who can apply standard ECG procedures from 12 to 50. Shimaa, an IPN trained nurse, stated, "Now we are able to discuss our needs with the head of the department. We can meet the hospital director and request supplies from the head nurse. We are now working as one team." This most recently trained group of nurses are applying their new skills in the emergency, internal medicine, surgery, orthopedics and urology departments of the hospital ${ }^{(19)}$.

The Improving Performance of Nurses (IPN) project started in Luxor International Hospital in November 2010. Twenty-eight nurses initially participated in MSH's Leadership Development Program (LDP) to develop their leadership and management skills, and worked in four teams to identify challenges affecting the quality of health services. They implemented action plans to address these challenges, and achieved the following results ${ }^{(18)}$ :

- The percentage of nurses working in operating theatres and surgical nephrology departments following MOH preoperative preparation standards increased by $49 \%$.

- Nurses who practiced proper cautionary measures while handling sharp instruments increased by $21 \%$.

- The percentage of nurses capable of properly separating and coding high risk medications increased by $78 \%$.

Nurse teams at all of the IPN supported hospitals continue to use the skills they learned in the LDP to improve performance in the departments where they work. Some critical care nurses also work with other hospitals to transfer the skills they have learned to additional nurses ${ }^{(17,19)}$.

\section{Conclusion}

In Egypt, Significant technological advances have allowed to develop sophisticated ventilators, renal replacement therapy and cardiovascular monitoring. Intensive care units can now even be supervised via tele or remote ICU systems, providing surveillance and support to a large number of ICUs in distant or remote sites by a centralized multidisciplinary critical care team.

\section{References}

[1]. Szmuk P, Ezri T, Evron S, Roth Y, Katz J. A brief history of tracheostomy and tracheal intubation, from the Bronze Age to the Space Age. Intensive Care Med. 2008 Feb;34(2):222-8.

[2]. Munro, Cindy L. The "Lady with the Lamp" Illuminates Critical Care Today. Am J Crit Care 2010; 19:315-317.

[3]. Adhikari NK, Fowler RA, Bhagwanjee S, Rubenfeld GD. Critical care and the global burden of critical illness in adults. Lancet. 2010 Oct 16;376(9749):1339-46.

[4]. McGaughey J, Alderdice F, Fowler R, Kapila A, Mayhew A, Moutray M. Outreach and Early Warning Systems (EWS) for the prevention of intensive care admission and death of critically ill adult patients on general hospital wards. Cochrane Database Syst Rev. 2007 Jul; (3) CD005529.

[5]. Jones D, George C, Hart GK, Bellomo R, Martin J. Introduction of medical emergency teams in Australia and New Zealand: a multi-centre study. Crit Care. 2008;12(2): R46. Epub 2008 Apr 7.

[6]. Mosallam R1, Hamidi S, Elrefaay M. Turnover intention among intensive care unit nurses in Alexandria, Egypt. J Egypt Public Health Assoc. 2015 Jun;90(2):46-51.

[7]. Pittard AJ. Out of our reach? Assessing the impact of introducing a critical care outreach service. Anaesthesia. 2003 Sep;58(9):882-5.

[8]. Bellomo R, Goldsmith D, Uchino S, Buckmaster J, Hart G, Opdam H, Silvester W, Doolan L, Gutteridge G. Prospective controlled trial of effect of medical emergency team on postoperative morbidity and mortality rates. Crit Care Med. 2004 Apr;32(4):916-21

[9]. Hillman K, Chen J, Cretikos M, Bellomo R, Brown D, Doig G, Finfer S, Flabouris A; Study investigators. Introduction of the medical emergency team (MET) system: a cluster-randomized controlled trial. Lancet. 2005 Jun 18 24;365(9477):2091-7.

[10]. Fletcher SJ, Cuthbertson BH. Outreach, epistemology and the evolution of critical care. Anesthesia. 2010 Feb;65(2):115-8

[11]. McDonnell A, Esmonde L, Morgan R, Brown R, Bray K, Parry G, Adam S,Sinclair R, Harvey S, Mays N, Rowan K. The provision of critical care outreach services in England: findings from a national survey. J Crit Care. 2007 Sep;22(3):212-8.

[12]. Esmonde L, McDonnell A, Ball C, Waskett C, Morgan R, Rashidian A, Bray K, Adam S, Harvey S. Investigating the effectiveness of critical care outreach services: a systematic review. Intensive Care Med. 2006 Nov;32(11):1713-21.

[13]. Priestley G, Watson W, Rashidian A, Mozley C, Russell D, Wilson J, Cope J, Hart D, Kay D, Cowley K, Pateraki J. Introducing Critical Care Outreach: a ward randomized trial of phased introduction in a general hospital. Intensive Care Med.2004 Jul;30(7):1398-404.

[14]. Peberdy M, Cretikos M, Abella B et al. Recommended guidelines for monitoring, reporting and conducting research on medical emergency team, outreach and rapid response systems. Resuscitation. 2007 75:412-433.

[15]. Funk D, Sebat F, Kumar A. A systems approach to the early recognition and rapid administration of best practice therapy in sepsis and septic shock. Curr Opin Crit Care. 2009 Aug;15(4):301-7.

[16]. Donald M. Berwick, MD, MPP, FRCP; David R. Calkins, MD, MPP; C. Joseph McCannon, BA; Andrew D. Hackbarth, BA. The 100000 Lives Campaign - Setting a Goal and a Deadline for Improving Health Care Quality. JAMA. 2006;295(3):324327. 
[17]. https://www.msh.org/our-work/projects/improving-the-performance-of-nurses-in-upper-egypt.

[18]. https://www.msh.org/our-work/country/egypt.

[19]. https://www.msh.org/our-work/health-system/leadership-management-governance. 